\title{
PDZ-based adaptor proteins in epithelial anion transport and VIP receptor regulation
}

\author{
Heon Yung Gee, Hyun Woo Park, Kyung Hwan Kim, and Min Goo Lee \\ Department of Pharmacology and Brain Korea 21 Project for Medical Science, Yonsei University \\ College of Medicine, Seoul, Korea
}

\begin{abstract}
Polarized protein deposition at the apical and basolateral membranes of epithelial cells is critical for the asymmetrical transport of ions and fluids across the epithelia. PDZ-based modular adaptor proteins are expressed in the junctional areas in epithelial cells and are generally part of a molecular scaffold that determines the localization and activity of ion channels, receptors, and other signaling molecules to their correct spatial arrangement for proper response to diverse stimuli. Hence, understanding the regulatory mechanisms of channels and receptors via PDZ-based adaptors will provide valuable insights into the physiology of epithelial cells as well as pathophysiology of many human diseases including cystic fibrosis. J. Med. Invest. 56 Suppl. : 302-305, December, 2009
\end{abstract}

Keywords : PDZ domain, CFTR, NHE3, VPAC, S-SCAM/MAGI-2

\section{PDZ-BASED ADAPTOR PROTEINS IN EPI- THELIAL TIGHT JUNCTIONS}

Specialized epithelial cells constitute selective barriers between diverse tissues and different body compartments in multicellular organisms. These cells are segregated into an apical and basolateral domain-that is, they are polarized-and they adhere to each other side-by-side through complexes that form junctions between the cells (1). These intercellular junctions play several important roles in the physiology of epithelial cells and tissues. First, they constitute a semipermeable barrier that controls the flow of ions and fluid through the paracellular pathway. Second, they contribute to the establishment and maintenance of the polarity of the apical and basolateral membranes. Third, they are the targets and effectors of diverse intracellular signaling pathways that control function, differentiation and proliferation

Received for publication November 10, 2009 ; accepted November 17, 2009 .

Address correspondence and reprint requests to Min Goo Lee, Department of Pharmacology, Yonsei University College of Medicine, 134 Sinchon-Dong, Seoul 120-752, Korea and Fax : +82-2313-1894. of epithelial cells. These cell junctions are assembled by an array of scaffolding molecules that link adhesion molecules and signaling proteins to the cytoskeleton (2). Genetic and biochemical studies over the past 20 years led us recognize the membrane-associated guanylate kinase (MAGUK) as ubiquitous scaffolding molecules concentrated at sites of junctional complexes. The MAGUKs contain several PDZ domains, one SH3 domain, and a guanylate kinase domain $(\mathrm{GuK})(3)$.

\section{PDZ DOMAINS}

PDZ domains were first discovered in three related MAGUKs including PSD-95, Dlg, and ZO1 , which were abbreviated by each initial letter. Genomic sequencing revealed that the $C$. elegans, Drosophila melanogaster, and Homo sapiens genomes each encode 60-100 PDZ proteins. The PDZ domain is a conserved protein-protein interaction module consisted of 80-90 amino acids which typically bind target proteins harboring specific C-terminal sequences called PDZ-binding motif (4). PDZ domains have two major functions. 1) They anchor 
integral membrane proteins including receptors, transporters, channels and adhesion proteins through their PDZ-binding motif and 2) they bind to the PDZ domains of other PDZ proteins, thus forming scaffolding networks mediated by homo- and heteromultimers. Therefore, PDZ domains cluster their target proteins in specific subcellular domains, determine the polarity of many proteins including channels and transporters and regulate their activity as well as endocytic trafficking. In addition, PDZcontaining proteins near the junctional area contain additional structural domains, which function as scaffolds for signaling proteins, such as small GTPases, kinases and transcription factors (5).

\section{REGULATION OF CFTR AND NHE3 BY PDZ-BASED PROTEINS}

The cystic fibrosis transmembrane conductance regulator (CFTR) localized at the apical membrane is a cyclic AMP/PKA-activated $\mathrm{Cl}^{-}$channel which functions as the key regulator of fluid and anion secretion in the gastrointestinal, respiratory, and genitourinary system. CFTR and other transporters, such as choloride-bicarbonate exchangers form large macromolecular complexes mediated by PDZbased adaptor proteins. Such adaptor proteins have been known to regulate CFTR activity in the plasma membrane. For example, EBP50/NHERF1 and E3KARP/NHERF2 augment cAMP-induced activation of CFTR by recruiting a cAMP-dependent protein kinase (PKA)/PKA-anchoring protein (AKAP) complex (6), whereas Shank2 attenuates the effect of cAMP on CFTR by placing the cyclic nucleotide phosphodiesterase PDE4D in proximity $(7,8)$. Conversely, in the case of $\mathrm{Na}^{+} / \mathrm{H}^{+}$exchanger 3 (NHE3) which plays a major role in $\mathrm{Na}^{+}, \mathrm{Cl}$ absorption in intestinal epithelia, EBP50/NHERF1 and E3KARP/ NHERF2 mediate cAMP-dependent inhibition of NHE3 (9), whereas Shank2 up-regulates its surface expression and basal activity (10). Therefore, NHERFs and Shank2 modulated CFTR and NHE3 in a reciprocal manner and by utilizing this combinatorial regulation, these adaptors can effectively govern the net epithelial transport via absorptive and secretory transporters (Fig. 1).

\section{REGULATION OF VIP RECEPTORS BY S- SCAM/MAGI-2}

Vasoactive intestinal peptide (VIP) is a 28 amino acid peptide that was first isolated in 1970 from the duodenum and lung. VIP is one of the major physiologic regulators of CFTR, which is released from

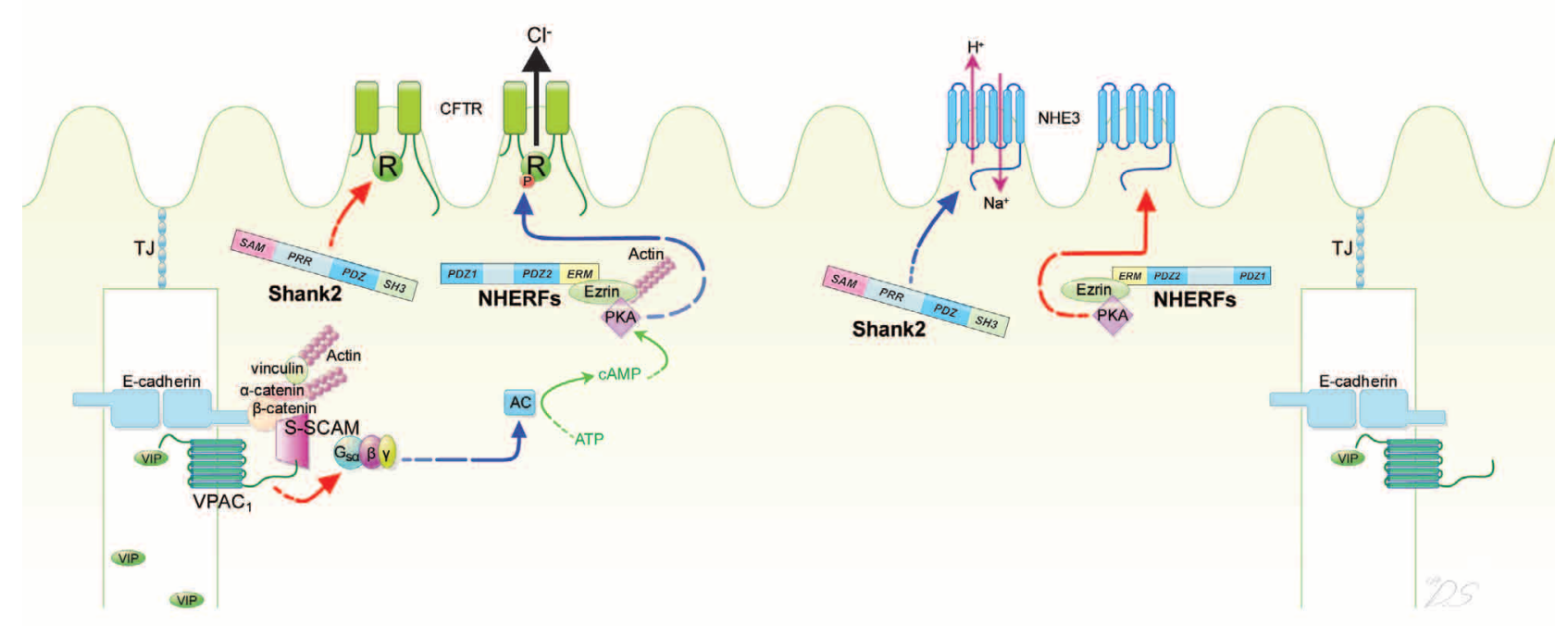

Fig. 1 Regulation of CFTR, NHE3 and VPAC1 by PDZ-based adaptor proteins in the plasma membrane of epithelial cells. The PDZ-based proteins NHERF1 and Shank2 regulate CFTR and NHE3 in an opposite manner. NHERF1 activates CFTR and inhibits NHE3, whereas Shank2 activates NHE3 and inhibits CFTR in epithelial cells (see text for details). Another PDZ scaffold, SSCAM/MAGI-2 recruits VPAC $_{1}$ to the junctional area and negatively regulates $\mathrm{VPAC}_{1}$ activity to prevent excess production of cAMP which can cause secretory diarrhea. The arrows indicate positive (blue) and negative (red) regulation. AC, adenylate cyclase; ERM, ezrin, radixin, moesin binding domain ; PDZ, PSD-95/Dlg/ZO-1 domain ; PRR, proline-rich region ; R, regulatory domain ; SAM, sterile $\alpha$ motif ; SH3, Src homology 3 domain ; TJ, tight junction. 
enteric nerves. Hence, it stimulates 1) biliary bicarbonate and water secretion from pancreatic and biliary duct cells, 2) enzyme secretion from pancreatic acinar cells, and 3) chloride secretion from intestinal epithelia. Such physiological actions of VIP are mediated via activation of two common G-protein coupled receptors $\left(\mathrm{VPAC}_{1}\right.$ and $\left.\mathrm{VPAC}_{2}\right)$ which preferentially stimulate adenylate cyclase followed by increased intracellular cAMP (11). Of those receptors, the $\mathrm{VPAC}_{1}$ receptor contains a typical PDZ-binding motif which makes it a strong candidate to interact with PDZ-based adaptors. Recently, we firstly reported the biochemical and functional interaction between $\mathrm{VPAC}_{1}$ and the synaptic scaffolding molecule (S-SCAM), a member of MAGUK PDZ-based scaffold, also known as membrane-associated guanylate kinase inverted-2 (MAGI-2) (Fig. 1) (12). S-SCAM/ MAGI-2 inhibited VIP-induced VPAC $_{1}$ internalization and cAMP generation which resulted in reduced CFTR $\mathrm{Cl}^{-}$current. Aberrant VPAC1 activation which causes the watery diarrhea syndrome in VIPoma (also known as Werner Morrison syndrome or WDHA syndrome) suggests that the delicate regulation of $\mathrm{VPAC}_{1}$ via S-SCAM/MAGI-2 is essential for VIP-mediated fluid and electrolyte secretion in normal epithelial cells. In addition, S-SCAM/ MAGI-2 associates with E-cadherin, a key protein at the adherens junction, in a $\beta$-catenin dependent manner (13) and recruits $\mathrm{VPAC}_{1}$ to the junctional area near the apical end of the lateral membrane in epithelial cells. Therefore, S-SCAM/MAGI-2 confines $\mathrm{VPAC}_{1}$ to the junctional area and restricts VIPinduced cAMP signaling within close proximity to the apical effectors such as CFTR. This, in turn, enables efficient electrolyte and fluid secretion in epithelial cells in response to VIP with minimal effects on the cell interior. This study demonstrates the critical role of PDZ-based scaffolds in receptormediated signal transduction as well as in the regulation of ion transporters and channels activity. It is noteworthy that PDZ-binding motifs are found in numerous receptors which imply the importance of these PDZ-based adaptor targets in wide range of receptor signaling (14). Emerging evidence suggests that PDZ-based adaptors utilize various modes of action to elicit effective hormonal response of physiological stimuli, such as 1) the assembly of signaling complexes which include hormone receptors and its effectors ; 2) the regulation of trafficking and tethering of membrane proteins, and 3) the modulation of second-messenger generation as described for $\mathrm{VPAC}_{1}$.

\section{CONCLUSIONS}

In conclusion, modulation of PDZ-mediated protein-protein interaction allows a single stimulus to result in diverse functional consequences. Hence, the balanced interactions between the membrane proteins including transporters, channels, and receptors and PDZ-based adaptors are likely to play a critical role in the homeostatic regulation of epithelial transport. Considering that both hypo- and hyper-functioning of receptors, channels or transporters may result in life-threatening diseases, such as cystic fibrosis or secretory diarrhea caused by CFTR, respectively, retaining prudent regulation via PDZ-based adaptor proteins is an important mechanism for the maintenance of body homeostasis.

\section{ACKNOWLEDGEMENTS}

We thank Dong-Su Jang for their editorial assistance. This work was supported by grants R01-2007000-20710-0 from the Korea Science and Engineering Foundation, and E00067 from the Korea Research Foundation, Ministry of Education, Science and Technology, Korea.

\section{REFERENCES}

1. Matter K, Balda MS : Signalling to and from tight junctions. Nat Rev Mol Cell Biol 4 : 225236, 2003

2. Guillemot L, Paschoud S, Pulimeno P, Foglia A, Citi S : The cytoplasmic plaque of tight junctions : a scaffolding and signalling center. Biochim Biophys Acta 1778 : 601-613, 2008

3. Funke L, Dakoji S, Bredt DS : Membrane-associated guanylate kinases regulate adhesion and plasticity at cell junctions. Annu Rev Biochem $74: 219-245,2005$

4. Kim E, Sheng M : PDZ domain proteins of synapses. Nat Rev Neurosci 5 : 771-781, 2004

5. Guggino WB, Stanton BA : New insights into cystic fibrosis : molecular switches that regulate CFTR. Nat Rev Mol Cell Biol 7 : 426-436, 2006

6. Yun CH, Lamprecht G, Forster DV, Sidor A : NHE3 kinase A regulatory protein E3KARP binds the epithelial brush border $\mathrm{Na}+\mathrm{H}+\mathrm{ex}-$ changer NHE3 and the cytoskeletal protein ezrin. J Biol Chem 273 : 25856-25863, 1998 
7. Kim JY, Han W, Namkung W, Lee JH, Kim $\mathrm{KH}$, Shin H, Kim E, Lee MG : Inhibitory regulation of cystic fibrosis transmembrane conductance regulator anion-transporting activities by Shank2. J Biol Chem 279 : 10389-10396, 2004

8. Lee JH, Richter W, Namkung W, Kim KH, Kim E, Conti M, Lee MG : Dynamic regulation of cystic fibrosis transmembrane conductance regulator by competitive interactions of molecular adaptors. J Biol Chem 282 : 10414-10422, 2007

9. Donowitz M, Cha B, Zachos NC, Brett CL, Sharma A, Tse CM, Li X : NHERF family and NHE3 regulation. J Physiol 567 : 3-11, 2005

10. Han W, Kim KH, Jo MJ, Lee JH, Yang J, Doctor RB, Moe OW, Lee J, Kim E, Lee MG : Shank2 associates with and regulates $\mathrm{Na}+\mathrm{H}+$ exchanger 3. J Biol Chem 281 : 1461-1469, 2006

11. Laburthe M, Couvineau A, Marie JC : VPAC receptors for VIP and PACAP. Receptors Channels $8: 137-153,2002$

12. Gee HY, Kim YW, Jo MJ, Namkung W, Kim JY, Park HW, Kim KS, Kim H, Baba A, Yang J, Kim E, Kim KH, Lee MG : Synaptic scaffolding molecule binds to and regulates vasoactive intestinal polypeptide type- 1 receptor in epithelial cells. Gastroenterology 137 : 607-617, 617 e601-604, 2009

13. Subauste MC, Nalbant $P$, Adamson ED, Hahn $\mathrm{KM}$ : Vinculin controls PTEN protein level by maintaining the interaction of the adherens junction protein beta-catenin with the scaffolding protein MAGI-2. J Biol Chem 280 : 56765681, 2005

14. Weinman EJ, Hall RA, Friedman PA, Liu-Chen LY, Shenolikar S : The association of NHERF adaptor proteins with $g$ protein-coupled receptors and receptor tyrosine kinases. Annu Rev Physiol 68 : 491-505, 2006 